\title{
Enfoques de Estudo de Alunos de Medicina
}

Dejano T. Sobral

\begin{abstract}
RESIJMC
Examina-se que falores afetam o enfoque de aprendizagem de alunos de Medicina, mediante a aplicação do Inventário de Enfoques de Estudo desenvolvido por Entwistle e colegas, o qual identifica três orientaçoses de estudo: significativa, reprodutiva e estratégica. Uma versāo abreviada do Inventário foi administrada a alunos de Medicina no inúcio do terceiro semestre e no decorrer do quinto semestre do curso. Foram obtidos dados de características dos estudantes, da avaliação inicial do curso e do rendimento académico no semestre da iniciação clínica, e medidas suas relações com os escores de orientạ̧ão de estudo. Os resultados revelam que a escala de orientaçāo significaliva mostra escores mais elevados que as demais, diferencia grupos (segundo sexo e nivel de motivação), apresenta associạ̧ão significante com a escala de orientação estratégica e com medidas de avaliação do curso e de rendimento acadêmico, e tem estabilidade moderada. As implicaçoses dos achados são comentadas. O perfil de orientação de estudo fornecido pelo Inventário utilizado pode ser útil para. monitorar as dimensōes de variação do enfoque de aprendizagem adotado por esludantes nos diversos contextos do ensino médico.
\end{abstract}

PAL AVRASCHAVI:

- líducagào Médica;

- Estudante de Medicina;

- Aprendivagem;

- Inventário de enfogues de estuda.

[REVISTA BRASI KIRA DHEADUCACAOMÉDIC

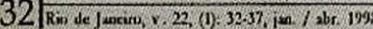

\section{INTRODUÇÃO}

Existem indicaçōes convincentes de que estudantes de nível universitário adotam diferentes enfoques de aprendizagem, os quais sāo determinados por características pessoais e fatores do contexto educacional. O conceito de enfoque de aprendizagem deriva dos estudos de Marton \& Saljo, que identificaram dois modos de processamento cognitivo, rotulados como profundo e superficial, os quais apresentam diferenças de propósito e processamento da informaçāo e levam a diferentes desfechos de aprendizado ${ }^{11}$. O enfoque profundo tende a produzir desfechos que representam um entendimento mais substancial da tarefa de aprendizagem, em razão do envolvimento pessoal e da busca do significado e de integraçāo do novo conhecimento ao conhecimento já adquirido. O enfoque superficial, diversamente, tende a produzir desfechos que representam baixo entendimento e caráter reprodutivo, em razāo de memorizaçāo desconexa de fatos e conceitos. 
O Inventário de Enfoques de Estudo, desenvolvido por Entwistle e colegas, incorpora esses dois enfoques de aprendizagem em suas múltiplas escalas ${ }^{7}$. Quatro orientaçōes de estudo foram identificadas nas investigaçōes realizadas: orientaçāo significativa (a forma operacional do enfoque profundo); orientaçāo reprodutiva (a forma operacional do enfoque superficial); orientaçāo estratégica (implicando intençāo estratégica); e orientaçāo não-acadêmica. Três versōes abreviadas do Inventário foram desenvolvidas, as quais incluem ao menos as escalas de orientaçāo significativa e de orientaçāo reprodutiva e contêm 18,30 ou 32 itens ${ }^{6,9,16}$.

Vários estudos utilizando uma versāo completa ou abreviada desse Inventário foram realizados com estudantes de diferentes cursos e países em uma ampla gama de situações, e seus resultados tendem a reforçar a credibilidade do instrumento na caracterizaçāo dos enfoques acima descritos. $\mathrm{O}$ uso do Inventário na área de Medicina, além de confirmar a distinção entre orientaçāo significativa e orientação reprodutiva, revelou três tipos de achado: (a) diferenças de escores de orientaçāo de estudo na comparaçāo entre grupos de estudantes de etapas diversas do mesmo curso, ou de cursos distintos com diferentes estratégias educativas; (b) associação entre escores de orientação de estudo e outras medidas de mesma perspectiva teórica; (c) tendência de associaçāo (nem sempre significante) entre perfis de orientaçāo de estudo e medidas de desempenho acadêmico ${ }^{3,4,10,14,21}$.

Este trabalho faz parte de uma análise da diferenciaçāo do enfoque de aprendizagem de estudantes de Medicina que tem o propúsito de identificar os fatores que intensificam ou reduzem a qualidade do aprendizado. Examinam-se aqui as orientaçōes de estudo caracterizadas pela aplicaçāo do formato abreviado do Inventário de Enfoques de Estudo proposto por Gibbs ${ }^{9}$ e tem três objetivos:

(1) identificar o perfil e a distribuiçāo sexual dos escores das orientações de estudo;

(2) estabelecer as relações dos escores de orientação com indicadores de motivaçāo e de autoconfiança, de avaliaçāo do curso e de rendimento acadêmico;

(3)definir as diferenças entre os escores de orientaçāo antes da iniciaçāo clínica e durante a mesma.

\section{MÉTODOS}

Plano

O estudo foi realizado em duas fases: na primeira, o Inventário foi aplicado à totalidade dos estudantes que completaram o primeiro ano do curso de Medicina na Universidade de Brasília; na segunda, o Inventário foi aplicado a uma parcela representativa do mesmo alunado durante a iniciaçāo clínica, cerca de 13 meses após a primeira aplicaçāo. Na primeira fase, o Inventário foi aplicado na primeira semana do terceiro semestre do curso, simultaneamente aos demais instrumentos. Na segunda fase, o Inventário foi aplicado na quinta semana do quinto semestre letivo para os alunos que cursavam uma disciplina optativa oferecida nesse semestre, o qual demarca a iniciaçāo clínica formal no currículo vigente.

\section{Participantes}

Na primeira fase, os participantes eram estudantes de turmas consecutivas do curso, os quais ingressaram na universidade a partir de 1994. O total geral alcançou 296 estudantes, a maioria $(59,8 \%)$ de sexo masculino. Na segunda fase, os participantes eram 186 alunos que alcançaram a fase clinica, 107 dos quais estaxam matriculados em turmas consecutivas de uma disciplina optativa, oferecida no quinto semestre do curso. Nessa amostra, 55,9\% dos estudantes eram do sexo masculino.

\section{Instrumentos}

O formato do Inventário utilizado nas duas fases do estudo contém 18 itens referentes a três de quatro escalas de orientação de estudo descritas por Entwistle ${ }^{6}$ :

a) orientaçāo significativa, caracterizada por busca de significado, uso de evidência, inter-relaçāo de idéias $e$ motivaçāo intrínseca;

b) orientaçāo reprodutiva, caracterizada por memorizaçāo, aprendizagem restrita e fatual, medo do fracasso e falta de conexāo de idéias;

c) orientaçāo estratégica, caracterizada por motivaçāo extrínseca, busca de requisitos externos e orientaçāo competitiva. 
O questionário utilizado tem escala de resposta de 5 pontos, de 4 (concordância definitiva) até 0 (discordância definitiva). As propriedades psicométricas do Inventário abreviado sāo descritas em recente relatório ${ }^{17}$.

No terceiro semestre, todos os participantes responderam também ao Inventário de Valorizaçāo do Curso (IVC), o qual é constituído de 36 itens distribuídos em quatro seçōes: valorizaçāo do curso, aprendizado de conteúdo, aprendizado pessoal e aprendizado de condutas. O IVC foi desenvolvido por Nehari e Bender ${ }^{13}$ para medir a apreciaçāo estudantil dos desfechos de aprendizado e serve como instrumento de avaliaçāo de curso $^{19}$. No caso, essa avaliaçāo teve como referência o primeiro ano do curso de Medicina. Em acréscimo, dois indicadores foram utilizados para descrever as características motivacionais dos aprendizes: motivaçāo para aprender e autoconfiança como aprendiz. Ambos foram medidos por escalas visuais de auto-relato, conforme consta em Sobral ${ }^{20}$.

$\mathrm{O}$ índice de rendimento acadêmico dos participantes foi calculado pela média ponderada das mençōes das disciplinas cursadas no quinto semestre do curso.

\section{Análise}

O trabalho de Andrews e colegas (1981) scrviu de guia na seleçāo de técnicas estatísticas ${ }^{1}$. Foram utilizados testes t para comparaçāo de médias de escores entre grupos e coeficientes de correlaçāo para aferir a associaçāo entre pares de medidas.

\section{RESULTADOS}

\section{Primeira fase}

As médias e os desvios padrōes (entre parênteses) das três escalas do inventário abreviado, para o total dos sujeitos, foram os seguintes: orientaçāo significativa $17,2(2,96)$; orientaçāo estratégica 16,8 $(3,30)$; orientaçāo reprodutiva $15,0(3,10)$. Usando o critério da pontuaçāo predominante, contrastando orientaçāo significativa e reprodutiva, $70,6 \%$ dos estudantes registraram um enfoque profundo e os restantes $29,4 \%$ mostraram um enfoque superficial de estudo (nota).

Dentre as escalas de orientaçāo, observou-se correlaçāo significante apenas entre os escores de orientaçāo significativa e de orientaçāo estratégica $(r=0,48)$. Os escores de duas escalas (orientaçāo significativa e orientaçāo estratégica) mostraram diferenças significantes entre estudantes de acordo com o sexo. A magnitude do efeito (effect size) nos escores a favor do sexo feminino foi moderada para a orientaçāo estratégica e pequena para a orientaçāo significativa. A Tabela 1 revela esses achados.

\section{TABELA 1}

Fscores médios (e desvios padrōes) de estudantes masculinos e femininos no Inventário de Fnfoques de Estudo ao término do primeiro ano do curso de Medicina

\begin{tabular}{|c|c|c|c|c|}
\hline $\begin{array}{l}\text { Orientaç̄o de } \\
\text { Estudo }\end{array}$ & $\begin{array}{c}\text { Dlasculinos } \\
n=177\end{array}$ & $\begin{array}{c}\text { Femininos } \\
n=119\end{array}$ & $p^{*}$ & Ml.1..t \\
\hline Significativa & $16.81(295)$ & $17,85(\overline{2,87})$ & 80,01 & 0.35 \\
\hline Reprodutiva & $15,00(3,3,3)$ & $15.06(2,74)$ & $>0,86$ & 0,02 \\
\hline Fstrategica & $16,13(3,44)$ & $17,85(2,82)$ & $\leqslant 0,001$ & 10,51 \\
\hline
\end{tabular}

* Significåncia das diferenças entre as médias.

\# Magnitude do efeito (effect size) = diferença cntre escores dividida por desvio padrão composto.

Foram observadas diferenças significantes nos escores de orientaçāo significativa e de orientaçāo estratégica de acordo com o nível de motivaçāo para aprender e de autoconfiança como aprendiz. Nos dois casos, escores de orientaçāo mais elevados estāo igualmente associados a alta motivaçāo e alta confiança (Tabela 2).

\section{TABELA 2}

Diferenças das médias de escalas de orientação de estudo entre estudantes com niveis baixo ou elevado de motivaçăo para aprender e de autoconfiançal como aprendiz

\begin{tabular}{|c|c|c|c|c|}
\hline Drientacto de & \multicolumn{2}{|c|}{ Motivacto para aprender } & \multicolumn{2}{|c|}{ dullonfinnce como aprendiz } \\
\hline Estudo & Baixu (n) $=1.32$ & Alto $(n=164)$ & Buixo $(n=87)$ & Allo $\{n=209$; \\
\hline Significativa & 16.311 & 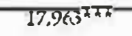 & $16,4<0$ & $17,545^{\pi \pi}-$ \\
\hline Reprodutiva & $15,08.3$ & $14,9 j 6 \mathrm{~ns}$ & 15.253 & $14,928 \mathrm{~ns}$ \\
\hline Estrattgica & $15,9.39$ & $17,537 * \star \star *$ & $15,6.55$ & $17,311^{\star \star \star \star}$ \\
\hline
\end{tabular}

ns $=$ nāo significante ** $p<0,01$

$\star \star \star ~ p<0,001$

A Tabela 3 mostra medidas de associaçāo entre os escores das três escalas de orientaçāo de estudo e o escore total do Inventário de Valorizaçāo do Curso. Correlaçōes significantes foram observadas entre a pontuaçāo da percepçāo do valor do aprendizado, referente ao primeiro ano do curso de Medicina, e os escores da escala de orientaçāo significativa $(r=0,44)$ e da escala de orientaçāo estratégica $(r=0,35)$.

TABEIAA 3

Correlaçōes entre escores de orientaçao de estudo e pontuação total do Inventário de Valorização do Curso $(\mathrm{N}=296)$

\begin{tabular}{|c|c|c|c|}
\hline \multirow{2}{*}{$\begin{array}{l}\text { Orientaçio de } \\
\text { Estudo }\end{array}$} & \multicolumn{3}{|c|}{ Inventário de $\nabla$ alorizaçăo de (.urso } \\
\hline & $\mathbf{r}$ & $t$ & $\mathrm{p}^{\star}$ \\
\hline Signilicativa & 0,44 & 8.4 & $<0,0001$ \\
\hline Reprodutiva & 0,03 & 0,5 & $>0,3$ \\
\hline Fstratćgica & 0,35 & 6,4 & $<0,0001$ \\
\hline
\end{tabular}

* Significância de coeficiente de correlação. 


\section{Segunda fase}

No conjunto dos estudantes, houve diferença entre as médias do índice de rendimento entre dois subgrupos: os estudantes com enfoque profundo apresentaram índice de rendimento acadêmico significantemente superior aos estudantes com enfoque superficial, no semestre de iniciaçāo clínica (Tabela 4).

\section{TABELA 4}

Médias e desvios padrōes do índice ck rendimento acadêmico em estudantes com enfoquc profuncks e enfoque superficial de aprendizagem $(N=186)$

\begin{tabular}{lccccc} 
Enfoque de & & \multicolumn{4}{c}{ Indice de Rendimento Académico } \\
Aprendizagem & $\mathrm{N}$ & Média & Desvio padrão & $p^{*}$ & M. F... it \\
\hline Tofundo & 1.30 & 3.903 & 0,526 & 0,015 & 0,43 \\
Superficial & 56 & .3 .659 & 0,586 & & \\
\hline
\end{tabular}

* Significância das diferenças entre as médias.

\# Magnitude do efeito (effect size) = diferença entre escores dividida por desvio padrāo composto.

A Tabela 5 mostra os dados de correlaçāo entre índice de rendimento acadêmico e escores de orientaçāo, na primeira e na segunda aplicaçāo do Inventário, para o subgrupo que cursou a disciplina optativa. Nāo foram observadas diferenças significantes, entre o subgrupo que cursou a disciplina optativa e o subgrupo controle, em relaçāo aos indicadores de características dos estudantes e de valorizaçāo do curso apurados no inicio do terceiro semestre.

\section{TABELA 5}

Correlaçoes entre escores de olientação de esludo, ma primcira e na segunda aplicaşăo, e índice de rendimento acadèmico $(\mathbb{N}=107)$

\begin{tabular}{|c|c|c|}
\hline \multirow{2}{*}{$\begin{array}{l}\text { Orientaçio te } \\
\text { Estudo }\end{array}$} & \multicolumn{2}{|c|}{ 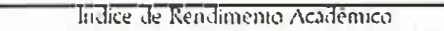 } \\
\hline & r (1'aplicaç;o) & r (2 aplicas.5o) \\
\hline Signilicstive & 0.25 & 0.17 \\
\hline Reprodutis. & 0.21 & 0,21 \\
\hline Estrateggicas & 0.23 & 0,24 \\
\hline
\end{tabular}

As correlações pareadas entre escores de orientaçāo de estudo na primeira e na segunda aplicaçāo do Inventário foram significantes para as três escalas: a correlaçāo mais forte foi registrada na escala de orientaçāo reprodutiva, e a mais fraca, na escala de orientaçāo significativa. A Tabela 6 mostra os escores de orientaçāo de estudo na segunda aplicaçāo do Inventário, feita no transcorrer do período letivo do quinto semestre do curso, comparados com os escores da primeira aplicaçāo. Foram observados declínios nos escores de orientaçāo significativa e de orientaçāo estratégica, enquanto houve elevação do escore de orientação reprodutiva. A extensāo das mudanças é realçada na comparação das proporções dos estudantes com enfoque profundo na primeira e na segunda aplicaçāo: houve queda de $69,2 \%$ para $38,3 \%$ na amostra do grupo eletivo.

\section{TABELA 6}

F.scores médios (desvios padrocs) na primeira e na segunda aplicaçāo do Inventário de Linfoques de Fistudo $(\mathrm{N}=107)$

\begin{tabular}{|c|c|c|c|c|}
\hline Orientaça de & \multicolumn{4}{|c|}{ Aṕlicaçāo do Invenţario } \\
\hline Estudo & Terceiro semestre. & Quinto semestre & 1 & $p^{*}$ \\
\hline Significativa & $16,841(2,901)$ & $15,037(3, \overline{383})$ & 4,18 & $<0,0001$ \\
\hline Reprodutiva & $15,075(2,708)$ & $1.5,927(3,548)$ & 1,97 & $<0,0.5 \#$ \\
\hline Estrategica & $16,897(3.025)$ & $15,174(3,407)$ & 3,91 & $<0,0001$ \\
\hline
\end{tabular}

* Significåncia de diferença entre médias.

\# teste $t$ de Welch.

\section{DISCUSSÃO}

A distinçāo entre diferentes enfoques de aprendizagem usados por estudantes no ensino superior parece ser uma característica universal que abrange os estudantes de Medicina. Admite-se atualmente que o enfoque adotado é uma resposta individual que depende das percepções dos estudantes em relaçāo aos conteúdos e às demandas das tarefas de aprendizagem, em um contexto particular ${ }^{5,15}$. Os achados deste trabalho ilustram essa noçāo.

As médias dos participantes nos escores das três escalas do Inventário sāo comparáveis aos valores referidos por Chessel, para estudantes de Aberdeen ${ }^{2}$. A exemplo de outros grupos de estudantes de Medicina, os participantes de Brasília registraram pontuaçāo mais elevada na orientaçāo significativa, que embute o conceito de enfoque profundo.

As diferenças entre homens e mulheres registradas nos escores de orientaçāo de estudo parecem peculiares. Estudos anteriores tendem a mostrar escores mais elevados de estudantes masculinos na orientaçāo significativa ${ }^{3,16,21}$. Evidências (nāo relatadas) sugerem que as diferenças entre sexos encontradas neste estudo, especialmente quanto aos escores de orientaçāo estratégica, têm relaçāo com características correlatas dos participantes que sāo pertinentes ao autocontrole da aprendizagem (responsabilidade e percepção de eficácia pessoal). A contribuiçāo da identidade sexual para a intensidade de orientaçāo de estudo (significativa ou estratégica) parece, por outro lado, independente dos efeitos do nível de motivaçāo para aprender e de autoconfiança como aprendiz. 
A relação entre a percepção do valor dos desfechos da aprendizagem (medido pelo IVC) e os escores de orientação significativa sugere uma interdependência entre características do contexto do curso $\mathrm{e}$ a intensidade da orientação adotada. Mitchel descreveu achados do mesmo teor e concluiu que os aprendizes que buscam refinar scu entendimento do que cstão aprendendo tendem a obter uma vivência de aprendizagem mais significativa e satisfatória ${ }^{12}$. Essa associação reforça a credibilidade do Inventário para monitorar mudanças da qualidade da aprendizagem, conforme relatado por Gibbs ${ }^{8}$.

Os dados da associação entre escores do Inventário e rendimento acadêmico são consistentes com outros estudos $\mathrm{cm}$ estudantes de Medicina, mas o sentido e o nível de correlação foram mais coerentes do que o geralmente relatado ${ }^{3,10,21}$. Possivelmente, o nível da associação entre rendimento e orientação de estudo relaciona-se com a capacidade de autocrítica e a percepção de auto-eficácia no controle da aprendizagem dos estudantes.

Escores mais elevados de orientação significativa no início do curso foram observados noutras escolas. Mas os resultados da segunda aplicação do Inventário surpreenderam pela extensão da queda da proporção de estudantes com enfoque profundo de aprendizagem e, eventualmente, da qualidade do aprendizado. Excluindo-se a menor adequação do instrumento ao contexto da aprendizagem clínica, as razōes para o fenômeno incluem os efe.tos da sobrecarga de conteúdos e tarefas e do próprio impacto emocional induzido pela iniciação clínica reduzindo a estabilidade dos procedimentos e do autocontrole do estudo. Dois fatos reforçam a noção da menor estabilidade da orientação significativa de estudo em condiçōes de pressão: o nível da correlação pareada e a queda da correlação com o índice de rendimento acadêmico, da primeira para a segunda aplicação do Inventário.

Os achados do estudo, em suma, têm implicaçōes para o acompanhamento do curso e realçam a importância da obtenção das perspectivas dos estudantes no processo de desenvolvimento educativo. Concluindo, o formato abreviado do Inventário de Enfoque de Estudo é um instrumento simples de aplicar e que pode ser útil para monitorar as dimensōes de orientação de estudo em diferentes contextos de aprendizagem, nos limites das qualidades psicométricas ${ }^{17}$. O Inventário, em circunstâncias cspeciais, pode servir também para ajudar os estudantes a refletir sobrc a própria concepção de aprendizagem e as habilidades corrclatas na preparação acadêmica ${ }^{18,21}$.

\section{Nota}

Os estudantes que registraram escore de orientação significativa superior ao escore de orientação reprodutiva foram classificados como tendo enfoque profundo de aprendizagem; na situação inversa, foram classificados como tendo enfoque superficial de aprendizagem. Na situação de empate nos escores, uma pontuação de orientação significativa superior a 17 levou à classificação de enfoque profundo e, em caso contrário, à classificação de enfoque superficial.

\section{SUMMARY}

Which learning approach is predominant among medical students? This article addresses the question by the use of the Approaches to Studying Inventory (ASI), developed by Entwistle et al. This questionnaire identifies three study orientations: meaning, reproductive, and strategic. A short version of ASI was administered to medical students at the start of the third term and during the fifth term of the medical program. Student descriptors and measures of course evaluation and student assessments were also obtained. The results show that the scale of meaning orientation has moderate stability and higher scores than the others. The scale differentiates between groups by sex and level of motivation and shows significant association with strategic orientation and measures of course evaluation and academic: achievement. In conclusion, the study orientation profile derived from ASI may be useful for monitoring the dimensions of variation of the learning approaches adopted by students in the different contexts of their medical education.

\section{KEXWORDS :}

- Education, medical;

-Student, medical;

- Learning;

- Approaches to studying inventory.

\section{REFERÊNCIAS BIBLIOGRÁFICAS}

1. ANDREWS, F. M., KLEM, L., DAVIDSON, T. N., O'MALLEY, P. M. \& ROGERS, W. L. A Guide for Selecting Statistical Techniques for Analyzing Social Science Data. Ann Arbor: ISR, University of Michigan, 1981. 
2. CHESSEL, G. Learning styles in first year medical students. Medical Tencher, v. 8, p. 125-135, 1986.

3. CLARKE, R. M. Students' approaches to learning in an innovative medical school: a cross-sectional study. Br.J. Educ. Psychol., v.56, p. 309-321, 1986.

4. COLES, C. Differences between conventional and problembased curricula in their students' approaches to studying Medical Education, v.19, p. 308-309, 1985.

5. ENTWISTLE, N. J. Reconstituting approaches to learning: a response to Webb. Higher Education, v. 33, p. 213-218, 1997.

6. ENTWISTLE, N. J. Styles of Learning and Tenching. Chichester: Wiley, 1981 .

7. ENTWISTLE, N. J., HANLEY, M. \& HOWNSELL, D. J. Identifying distinctive approaches to studying. Higher Education, v. 8, p. 365-380, 1979.

8. GIBBS, G. Improving the Quality of Student Learning. Bristol: Technical \& Educational Services, 1992.

9. GIBBS, G., I-IABESI-IAW, S. \& I-IABESHAW, T. 53 Interesting Ways to Appraise Your Tenching. Bristol: Technical \& Educational Services, 1988.

10. LEIDEN, L.I., CROSBY, R.D. \& FOLLMER, H. Assessing learning styles inventories and how well they predict academic performance. Academic Medicine, 65: 395-401, 1990.

11. MARTON, F. \& SALJO, R. On qualitative differences in learning. I - Outcome and process.Br.J. Educ. Psychol., 46: 4-11, 1976.

12. MITCHEILL, R. The development of the cognitive behavior survey to assess medical student learning. Teaching and Learning in Medicine, 6: 161-167, 1994.

13. NEHARI, M. \& BENDER, H. Meaningfulness of a learning experience: a measure for educational outcomes in higher education. Higher Education, 7: 1-11, 1978.

14. NEWBLE, D. I. \& CLARKE, R. M. The approaches to learning of students in a traditional and in an innovative problembased school. Medical Edilcation, 20: 267-273, 1986.

15. RICHARDSON, J. T. E. Using questionnaires to evaluate student learning: some health warnings. In G. Gibbs (ed.) Improving Student Learning. Theory and Practice. Oxford: Oxford Centre for Staff Development, 1994.
16. RICHARDSON, J. T. E. Gender differences in responses to the Approaches to Studying Inventory. Studies in Higher Education, v. 18, p. 3-13, 1993.

17. SOBRAL, D.T. Análise psicométrica de dois formatos abreviados do Inventário de Enfoques de Estudo com respondentes do curso de Medicina. Relatório preliminar. Brasília, Universidade de Brasília, 1997.

18. SOBRAL, D.T. Improving learning skills: a self-help group approach. Higher Education, v. 33, p.39-50, 1997.

19. SOBRAL, D. T. Efeitos da mudança curricular: características do aprendiz e desfechos da aprendizagem. R. Bras Educ. Méd., v. 20, p.15-20, 1996.

20. SOBRAL, D. T. Motivaçāo para aprender e resultados da aprendizagem baseada em problemas. Psicologin: Teorin e Pesquisa, v. 9, p.557-564, 1993.

21. STIERNBORG, M. \& BANDARANAYAKE, R. C. Medical students' approaches to studying. Medical Tencher, v. 18, p 229-236, 1996

\section{Endereço para correspondência:}

Universidade de Brasília - Faculdade de Ciências da Saúde

Caixa Postal 15-3031

70910-900 - Brasília - DF

\section{ERRATA}

$O$ artigo Enfoques de estudo de alunos de Medicina do Prof. Dejano T. Sobral, publicado no volume 22, número 1 , saiu com dois erros de impressão à página 35 . Pedimos desculpas ao autor e aos leitores.

TABELA 5. Correlações entre escores de orientação de estudo, na primeira e na segunda aplicação, e índice de rendimento acadêmico $(\mathrm{N}=107)$

\begin{tabular}{|c|c|c|}
\hline Orientação de Estudo & \multicolumn{2}{|c|}{ Índice de Rendimento Acadêmico } \\
\hline & $\mathrm{R}\left(1^{\mathrm{a}}\right.$ aplicação) & $\mathrm{R}\left(2^{\mathrm{a}}\right.$ aplicação) \\
\hline Significativa & 0,25 & 0,17 \\
\hline Reprodutiva & $-0,21$ & $-0,21$ \\
\hline Estratégica & 0,23 & 0,23 \\
\hline
\end{tabular}

TABELA 6. Escores médios (desvios padrões) na primeira e na segunda aplicação do Inventário de Enfoques de Estudos ( $\mathrm{N}=107)$

\begin{tabular}{|c|c|c|c|c|}
\hline Orientação de Estudo & \multicolumn{5}{|c|}{ Índice de Rendimento Acadêmico } \\
\hline & Terceiro semestre & Quinto semestre & $\mathrm{T}$ & $\mathrm{P}^{*}$ \\
\hline Significativa & $16,841(2,901)$ & $15,037(3,383)$ & 4,18 & $<0,0001$ \\
\hline Reprodutiva & $15,075(2,708)$ & $15,927(3,548)$ & 1,97 & $<0,05$ \\
\hline Estratégica & $16,897(3,025)$ & $15,174(3,407)$ & 3,91 & $<0,0001$ \\
\hline
\end{tabular}

